Editorial

\title{
A Look Inside of the Complex Pathogenesis of B-RAF(V600E)-Driven Cancer
}

\author{
Emilia Caputo $\bowtie$ \\ Institute of Genetics and Biophysics -I.G.B., A. Buzzati-Traverso-, CNR, Via Pietro Castellino, 111, I-80131 Naples, Italy. \\ $\square$ Corresponding author: Dr. E. Caputo, Institute of Genetics and Biophysics -I.G.B., A. Buzzati-Traverso-, CNR, Via Pietro Castellino, 111, I-80131 Naples, \\ Italy; Tel. +39 081 6132307; Fax: +39 081 6132706; E-mail: emilia.caputo@igb.cnr.it \\ (c) Ivyspring International Publisher. This is an open access article distributed under the terms of the Creative Commons Attribution (CC BY-NC) license \\ (https://creativecommons.org/licenses/by-nc/4.0/). See http://ivyspring.com/terms for full terms and conditions.
}

Published: 2017.05.26

\begin{abstract}
Cancer is a very complex disease, where cell alterations (i.e. gene mutations, dysregulated signaling pathway(s) and epigenetic modifications) contribute to its development and progression. Elucidating the connections among these cell modifications is a critical challenge, in order to understand the mechanisms which are activated from cancer cells to survive and proliferate. Interesting, Prof. Peng Hou and colleagues, at the First Affiliated Hospital of Xi'an Jiaotong University in China, identified unknown epigenetic mechanisms and their involvement in the tumorigenesis of B-RAF(V600E)-driven cancer.
\end{abstract}

Recently growing attention has been placed on mutations in B-RAF gene, encoding for a serine-threonine kinase, involved in RAS/RAF/MEK/ERK Mitogen Activated Protein Kinases (MAPK) pathway. Among the different mutations $(\sim 40)$ identified in B-RAF gene, the more frequent, B-RAF(V600E), has been found in the exon 15 and it resulted in the substitution of valine for glutamic acid at position 600 of the BRAF protein. This is an activating mutation, responsible for activating constitutively the MAPK pathway, promoting cell proliferation and preventing apoptosis. It is mostly found in human cancer such as papillary thyroid cancer (PTC), melanoma and metastatic colon cancer (1).

Based on these data, therapeutic strategies have been developed to treat cancer patients with specific inhibitors of the mutated oncogenic protein B-RAF(V600E), such as vemurafenib and dabrafenib. B-RAF oncogenic inhibitor-based therapy produced tumor responses in the majority of patients and it prolonged median survival compared to chemotherapy. However, responses were largely partial, and clinical evidences of tumor resistance were observed, due to the activation of adaptive mechanisms switched on from cancer cells upon the drug selective pressure as well as consequence of the intrinsic tumor cell plasticity potential $(2,3)$.

Genetic alterations and dysregulation of signaling pathway(s) are not the only cell modifications defining the driving force of tumorigenesis and cancer progressions. Epigenetic modifications have been also reported to contribute to the very complex pathogenesis and pathophysiology of cancer. Epigenetic changes are defined as heritable and reversible changes in gene activity and expression, occurring without changes in DNA sequence. These non-genetic alterations are tightly regulated by two major epigenetic modifications: DNA methylation and histone modifications. However, it is becoming more evident that epigenetic mechanisms include also small and non-coding RNAs, and chromatin architecture (4).

Interesting, a recent study by Prof. Peng Hou and colleagues at the First Affiliated Hospital of Xi'an Jiaotong University in China, highlights unknown epigenetic mechanisms, involved in the tumorigenesis of B-RAF(V600E)-driven cancer (5). Prof. Hou showed the connection between B-RAF(V600E) mutation and the trimethylation of the histone, H3K27. This histone 
is known for shutting down transcription and, thereby, for participating in the control of gene expression patterns. H3K27 is tri-methylated (H3K27me3) on lysine in position 27 from the Enhancer of Zeste (EZH) 2 methyl-transferase, a component of the polycomb repressive complex 2 (PRC2). EZH2 is responsible for the repression of many genes involved in development, cell differentiation and cancer, by the epigenetic maintenance of the H3K27me3 repressive chromatin mark.

The investigators explored if the B-RAF(V600E) signaling was associated with the H3K27me3 epigenetic modification. To this end, they generated NIH3T3 cells expressing human B-RAF(V600E) and B-RAF wild-type, by transfecting them with vector containing the mutated and the wild-type B-RAF human gene, respectively and with the empty vector as experimental control. They observed that the NIH3T3 cells carrying B-RAF mutation showed an increase of Erk1/2 phosphorylation compared to the NIH3T3 B-RAFwt and to experimental control, as expected, being B-RAF(V600E), involved in the constitutive activation of MAPK signaling pathway. Interestingly, they observed in the NIH3T3 B-RAF(V600E) a significant increase in the level of H3K27me3 compared to the B-RAFwt and to experimental control, suggesting that the involvement of activated MAPK signaling in the H3K27me3 epigenetic modification. Same results were also obtained in human cancer cell lines, carrying B-RAF(V600E) mutation, derived from melanoma and papillary thyroid cancer.

Furthermore, Prof. Hou and colleagues explored the H3K27me3-enriched targets in the NIH3T3 cells carrying B-RAF mutation and compared them to the ones obtained from the NIH3T3 B-RAFwt cells, by performing ChIP-Seq experiments. At the same time, they performed gene expression analysis in both the cell lines (NIH3T3 B-RAF(V600E) and NIH3T3 $\mathrm{B}-\mathrm{RAF}^{\mathrm{wt}}$ ) and integrated these gene expression data with the ChIP-Seq results. They found that 150 down-regulated genes exhibited higher enrichments of H3K27me3 around the transcriptional start site (TSS) regions in the NIH3T3 cells carrying B-RAF mutation versus the NIH3T3 B-RAFwt cells, further supporting the shutting down transcription role of H3K27me3.

These data were validated 'in vitro', by ChIP-qPCR and qRT-PCR, choosing randomly 8 genes among the 150 identified. They were also validated 'in vivo'. In particular, they used an 'in vivo' system consisting of transgenic mouse lines (TPO-Cre and B-RAFCA), able to develop papillary thyroid cancer carrying B-RAF(V600E) mutation. In order to further confirm the role of B-RAF (V600E) in the regulation of these genes, the mice were treated with B-RAF inhibitors alone or in combination with MEK-inhibitors, and in both cases the investigators found that the expression of these downregulated genes was restored. Thus, these findings supported the role of the B-RAF signaling in the silencing of some genes, during the tumor progression, by H3K27me3 epigenetic modification.

Furthermore, Prof. Hou and colleagues highlighted the way B-RAF signaling was connected to the trimethylation of H3K27. As previously mentioned, H3K27 is tri-methylated (H3K27me3) on lysine in position 27 from the EZH2 methyl-transferase, a component of PRC2. This complex has been reported to be regulated by c-myc, a downstream factor of B-RAF activated signaling. Thus, the investigators hypothesized a connection between B-RAF signaling and H3K27 trimethylation through c-myc protein. They elegantly demonstrated their hypothesis by performing 'in vitro' and 'in vivo' experiments. They observed that the c-myc knockdown in NIH3T3 cells induced an inhibition of basal level expression of the components of the PRC2 (Ezh2, Suz12 and Jarid2) as well as H3K27me3. Same results were observed 'in vivo' by treating the mice with 10058-F4, a c-myc inhibitor. In addition, the investigators found that the expression of the 8 validated downregulated genes was restored in the mice treated with 10058-F4 agent. Same results were obtained in human cancer cell lines carrying B-RAF(V600E) mutation, supporting furthermore the connection between B-RAF mutation and H3K27 tri-methylation through c-myc factor.

It has been previously reported that c-myc plays a critical role by integrating regulatory networks involved in the maintenance of pluripotency and in cell programming. In this context, it has been demonstrated that most of developmental-related genes, involved in multi-lineage commitment, were down-regulated by c-myc and were targeted by PRC2 and/or PRC1 complex. For some of these down-regulated genes, it has been also demonstrated a direct role of c-myc in the recruitment of PRC2 complex on their promoters, to induce their transcriptional repression. In addition to this myc-mediated direct modulation, it has been demonstrated an indirect c-myc role, through a regulatory circuitry including multiple mi-RNAs.

The authors investigated the mechanism by which c-myc was involved in the maintenance of gene silencing in B-RAF(V600E)-driven cancer. The investigators demonstrated that c-myc was involved in the H3K27me3 epigenetic modification, through regulating the components of the PRC2 complex at 
transcriptional levels, by direct binding to their regulatory elements, as well as at post-transcriptional level, by repressing the miR-26a, miR-200b and miR-155 expression. They demonstrated that c-myc was essential in the down-regulation of miR-26a, miR-200b and miR-155 with consequent up-regulation of the components of the PRC2 (Ezh2, Suz12 and Jarid2) and H3K27me3 epigenetic modification. They also showed that B-RAF(V600E) mutation was able to induce silencing by affecting the chromatin architecture, through Erk1/2-induced RNA polymerase II (RNAPII).

This study supports the growing evidences that genetic and epigenetic alterations in cancer allow the tumor cells to modulate complex regulatory networks, in order to survive and proliferate. Prof. Hou contributes to the identification of epigenetic modifications induced in cancer cells after their acquisition of B-RAF(V600E) mutation. Furthermore, his study provides to the scientific community, ChIPseq and gene expression analysis data, which can be explored to improve our knowledge on tumor biology, keeping in mind their limitations being them obtained in a mouse background. Thus, it will be critical to perform ChIP-seq and gene expression analysis experiments on human cancer cell lines, with or without B-RAF mutation, and compare these data with those obtained from NIH3T3 cell system, in order to provide useful information for the development of therapeutic strategies and diagnostic tools in human cancer. Even better it would be to perform the same experiments on human tissue specimens from a large number of cancer patients as well as from the same patient during cancer evolution. However, these studies are limited from the tumor tissue availability and from the lack of techniques able to detect and capture enough cancer cells from patients during the dormancy phase of tumor progression, to further study their molecular properties.

Despite of the increasing body of genetic and epigenetic alterations identified in cancer, the efficacy of drug agents targeting these alterations remains limited, likely due to the intrinsic molecular heterogeneity of cancer and subsequent drug resistance development. In conclusion, it is certainly critical to investigate the connections among the networks used from cancer cells to get more inside of tumor biology, but it is challenging to study how these complex networks are dependent on the microenvironment and on the specific selective pressure during cancer progression, thus taking into account the tumor cell heterogeneity and plasticity.

\section{References}

1. Asati V, Bharti SK, Mahapatra DK. Mutant B-Raf Kinase Inhibitors as Anticancer Agents. Anticancer Agents Med Chem. 2016; 16: 1558-75.

2. Sun C, Wang L, Huang S, et al. Reversible and adaptive resistance to BRAF(V600E) inhibition in melanoma. Nature 2014; 508: 118-22.

3. Cordaro FG, De Presbiteris AL, Camerlingo R, et al. Phenotype Characterization of Human Melanoma Cells Resistant to Dabrafenib. Oncology Reports; in press.

4. Perri F, Longo F, Giuliano M, et al. Epigenetic control of gene expression: Potential implications for cancer treatment. Crit Rev Oncol Hematol. 2017; 111: 166-72.

5. Qu Y, Yang Q, Liu J, et al. c-Myc is Required for BRAFv600E-Induced Epigenetic Silencing by H3K27me3 in Tumorigenesis. Theranostics 2017; 7(8):2092-2107. doi:10.7150/thno.19884. 